\title{
Impacto del sistema de alertas "semáforo" de contenido de azúcar, sal y grasa en etiquetas de alimentos procesados: enfoque cualitativo
}

\author{
Impact of sugar, salt and fat warning labels \\ in processed food: A qualitative approach
}

Antonio Francisco Poveda Guevara ${ }^{1}$

directormarketing@uteg.edu.ec

\begin{abstract}
Resumen
El Ministerio de Salud Pública de Ecuador (LATAM), por decreto ministerial implementó en noviembre de 2013 una medida innovadora en un esfuerzo por mejorar los hábitos alimenticios de la población ecuatoriana y así reducir la incidencia de enfermedades causadas por una nutrición deficiente. Los productores de alimentos procesados se vieron obligados a poner en sus etiquetas de producto un sistema gráfico que indique a los consumidores la cantidad de grasa, azúcar y sal en sus productos mediante el uso de colores rojo, amarillo y verde como advertencia. Esta investigación busca analizar el impacto de la implementación de este sistema de alerta en el consumo de alimentos procesados para considerar si la medida realmente ha tenido el impacto deseado y ha reducido el consumo de productos con altos niveles de azúcar, sal y grasa. A través de entrevistas en profundidad con jefes de hogar de clase media y media alta de la ciudad de Guayaquil, se encontró que en general los ciudadanos están de acuerdo con la medida y consideran negativamente productos con alerta roja (alto contenido de azúcar, sal y grasa). A pesar de que han reducido la cantidad de consumo de este tipo de productos, son pesimistas sobre la posibilidad de dejar de comprarlos.
\end{abstract}

\section{Palabras clave}

Embalaje, alimento, política de la salud, nutrición, azúcar, sal, caloría, dietética.

Forma sugerida de citar: Poveda Guevara, Antonio Francisco (2016). Impacto del sistema de alertas "semáforo" de contenido de azúcar, sal y grasa en etiquetas de alimentos procesados: enfoque cualitativo. Universitas, XIV(25), pp. 48-60.

1 Universidad Tecnológica Empresarial de Guayaquil-UTEG. Candidato a doctor en Gestión Económica Global (2012-2016) por la Universidad Nacional Mayor de San Marcos (Lima, Perú). Magister en Administración de Empresas por la universidad Carlos III de Madrid. Candidato a Magister en Marketing (2014-2016) por la Universidad de especialidades Espíritu Santo (Guayaquil, Ecuador). Director de la carrera de Gestión Empresarial mención Marketing y Ventas de la Universidad Tecnológica Empresarial de Guayaquil. 


\begin{abstract}
The Ministry of Public Health of Ecuador (LATAM), by ministerial decree implemented in November 2013 an innovative measure in an effort to improve the eating habits of the Ecuadorian population and thus reduce the incidence of diseases caused by poor nutrition. The producers of processed foods were obliged to put on their product labels a graphic system that indicates consumers the amount of fat, sugar and salt in their products by using colors red, yellow and green as a warning. This research seeks to analyze the impact of the implementation of this warning system in the consumption of processed foods to consider whether the measure has actually had the desired impact and has reduced the consumption of products with high levels of sugar, salt and fat. Through in depth interviews with middle and upper middle class heads of household in the city of Guayaquil, it was found that in general the citizens agree with the measure and regard negatively products with red alert (high sugar, salt and fat). Even though they have reduced the amount of consumption of this type of products, they are pessimistic about the possibility of completely stop buying them.
\end{abstract}

Keywords

Packaging, food, health policy, nutrition, sugar, salt, calories, dietetics.

\title{
Introducción
}

Ecuador se ha convertido en el primer país en Latinoamérica en reglamentar la obligatoriedad de la colocación de un sistema de semáforo en las etiquetas de productos alimenticios para alertar a los consumidores sobre la cantidad de azúcar, sal y grasa (Ortiz, 2014). La medida tomada en el acuerdo ministerial 4522 del 15 Noviembre del 2013 contiene el reglamento sanitario de etiquetado de alimentos procesados para el consumo humano cuyo objetivo principal es regular y controlar el etiquetado de alimentos procesados garantizando información oportuna, clara, precisa y no engañosa sobre el contenido de los alimentos (Ministerio de Salud, 2013). El Artículo 12 del reglamento establece que se ubicará en las etiquetas de los productos un sistema gráfico con barras de colores rojo, amarillo y verde que reflejaran el nivel de concentración de azúcar, sal y grasa de bajo 3 categorías: de concentración "Baja", de concentración "media", de concentración "alta". 
Este sistema debía estar enmarcado en un cuadro de fondo gris o blanco en la parte frontal de la etiqueta. Sin embargo, posteriormente el Acuerdo Ministerial 4866 permitió que pueda ser ubicado en el panel secundario (parte de atrás) lo cual demuestra que de alguna manera el Ministerio de Industrias flexibilizó las reglas (Orozco, 2014).

El "Reglamento sanitario sustitutivo de etiquetado de alimentos procesados para el consumo humano" (Ministerio de Salud Pública, 2014) publicado el lunes 25 de Agosto, contempla cambios interesantes, entre ellos la modificación de la prohibición de usar logos, certificaciones y/o sellos de asociaciones, sociedades, fundaciones, federaciones o de grupos colegiados, que hagan referencia a la calidad y composición del producto. En su lugar se indica que estos únicamente no pueden hacer referencia a beneficios a la salud por el consumo de un determinado alimento procesado. Se elimina también la prohibición de usar la imagen de profesionales de la salud y celebridades. Pese a que la colocación de "semáforos" indicadores en las etiquetas de productos alimenticios no se ha realizado en muchos países, existen varios artículos sobre esta temática. A continuación se muestra algunos resultados de los estudios presentados por diversos autores:

En su review, Saenz-Valero (2012) indica que fue capaz de encontrar 133 artículos mediante revisión de la literatura científica sobre las intervenciones orientadas a reducir el consumo de sal a través de mensajes en el etiquetado.

Ayala et al. (2010), a través de una encuesta por correo en los años 2005 y 2008 , encuentra que en Estados Unidos se redujo el consumo de sal de $48 \%$ a $56 \%$ y aumentó la lectura del contenido de las etiquetas de $64 \%$ a $69 \%$. Algunos años antes, Heimbach (1985) había realizado una encuesta en el citado país encontrando que el $38 \%$ de los encuestados dijeron leer las etiquetas de los productos para evitar consumir sal en exceso. Adicionalmente, en el mencionado estudio se consulta a los encuestados las causas por las cuales consideran ellos que las personas no optan por productos bajos en sal y el $49 \%$ indicó como motivo que los productos bajos en sal son más caros; $38 \%$ debido a que no tienen buen sabor y $35 \%$ porque consideraban que eran productos para personas enfermas.

Según Grimes et al. (2009) en un estudio en centros comerciales de Melbourne- Australia el 69\% de los encuestados dijo leer el etiquetado de sal a la hora de comprar pero preocupantemente el $50 \%$ de la muestra no fue capaz de realizar compras bajas en sal, a pesar de apoyarse en la etiqueta. 
Otro estudio en Australia concluyó que el uso de semáforo de advertencia frontal hacía que fuese cinco veces más probable que los entrevistados identificaran productos más saludables que con otro tipo de etiquetas (Kelly et al., 2009).

Feunekes et al. (2008) analizó la aplicación del sistema semáforo en la etiqueta frontal de tres productos a través de encuesta en cuatro países europeos. Considerando dimensiones tales como el gusto (liking), comprensión, credibilidad y salud percibida; encontró que el sistema de etiquetado es entendido, gustado y creíble pero inferior a otros sistemas para diferenciar productos saludables de los no tan saludables.

Sin embargo, Mhurchu \& Gorton (2007), encuentran que los consumidores no son capaces de comprender etiquetas que muestran diferentes nutrientes simultáneamente, por lo que terminan basando su decisión de compra únicamente en función del contenido de grasa.

A partir de la consulta de los resultados de las investigaciones mencionadas anteriormente, este trabajo pretende analizar cuál es el impacto en el consumo alimenticio de la familia guayaquileña ocasionado por la colocación del sistema gráfico de alerta del nivel de grasas, azucares y sal "semáforo" en las etiquetas de productos alimenticios procesados. El investigador aborda el problema de investigación a través del análisis de 4 subpreguntas de investigación que permiten un exhaustivo análisis del impacto en el consumo del sistema de alertas en el etiquetado:

- ¿Cuál es el proceso de compra que llevan a cabo las familias guayaquileñas en el supermercado?

- ¿Cuáles son los factores que afectan la decisión de compra en el supermercado?

- ¿Se percatan o son conscientes los consumidores de la existencia del sistema gráfico en los productos que consumen?

- ¿ ¿La familia guayaquileña considera beneficioso o útil para la salud la presencia del sistema gráfico "semáforo" en los productos?

\section{Materiales y métodos}

La presente investigación se llevó a cabo a través de entrevistas a profundidad a veinte padres/ madres de familia, recopilando exclusivamente la opinión del jefe del hogar que asiste al supermercado a realizar las compras familiares. 
En este proceso de diagnóstico el autor toma una posición neutra y objetiva, buscando conocer la apreciación real de los entrevistados sin influir de alguna manera en sus respuestas realizando las entrevistas en el hogar de los participantes previo su consentimiento.

No se solicitó datos personales ni se realizaron grabaciones para no intimidar a los participantes.

La entrevista fue semiestructurada, el investigador contó con una guía con los temas a abordar de acuerdo a los objetivos de la investigación, pero permitió al entrevistado profundizar en opiniones fuera de las preguntas planificadas cuando consideraba que la información proporcionada era de interés para la investigación.

El estudio se realiza únicamente en la ciudad de Guayaquil a través de la selección de una muestra tomada por conveniencia y a criterio del investigador considera por tanto la opinión de un limitado grupo de jefes de hogar (adultos con hijos) de clase media y media alta con estudios de tercer nivel concluidos, en su mayoría a padres de familia de estudiantes de una universidad privada de la ciudad de Guayaquil.

\section{Resultados}

\section{El proceso de compra en supermercados $y$ factores que lo afectan}

En base al análisis de las diferentes respuestas y la agrupación de opiniones similares, fue posible definir tres procesos de compra distintos los cuales se relacionan a continuación:

\section{Proceso de compra no meditado}

Este proceso corresponde al que realizan los consumidores que al asistir al supermercado adquieren siempre los mismos productos y marcas por lo que no se fijan en los precios o la calidad. Tampoco realizan ningún tipo de comparación o evaluación de beneficios entre las distintas marcas, siendo por tanto los consumidores más leales.

Cuando voy al súper ya sé que marcas me gustan y las que les gustan a mis hijos y voy directo a buscarlas (Madre de familia). 
En este proceso de compra, los hijos suelen tener alta influencia en la decisión de compra ya que los padres adquieren sin realizar ningún análisis los productos que saben que sus hijos prefieren.

En alguna ocasión me pasó que no había la leche que mi hijo toma y llevé otra pero, no se la tomó y terminé botándola. Mejor comprar lo que les gusta (Padre de familia).

\section{Proceso de compra meditado y analítico}

Un grupo mayoritario de los consumidores manifestó que si realizaba una evaluación y comparación de los productos en el punto de venta para poder decidir cuál adquirir. Estos consumidores analizan si comprar marcas establecidas, desconocidas o marca del supermercado en base a su evaluación de la calidad, sabor y calorías.

Si bien estos compradores comparten el nivel de evaluación de los productos, el peso que cada uno de ellos lo otorga a cada factor puede ser muy diferente. En su mayoría los consumidores dan un gran peso de importancia al precio y luego analizan el resto de variables.

... por la situación uno siempre anda buscando el ahorro. Comparo precios y sacrifico muchas veces no llevar las marcas conocidas para poder comprar más (Madre de familia entrevistada).

\section{Proceso de compra crítico y saludable}

El último grupo de análisis refleja un proceso de compra sumamente crítico que valora la calidad y salud por sobre cualquier otro factor. Este consumidor manifiesta que se toma su tiempo para adquirir los productos comparando y analizando las diversas opciones disponibles en el supermercado prefiriendo siempre los alimentos orgánicos, frescos, saludables y nutritivos.

Mis hijos llegaron a decirme que por qué no podía ser una "madre normal" y comprar cualquier producto sin analizarlo tanto, pero es que es importante comprar alimentos que no sean dañinos y que no afecten nuestra salud (Madre de familia).

Los resultados obtenidos en cuanto al proceso de compra y los factores que lo afectan se resumen en la siguiente tabla: 
Tabla 1

Procesos de compra y factores que afectan la compra

\begin{tabular}{|c|c|c|}
\hline Proceso de compra & Tipo de consumidor & Factores que afectan la compra \\
\hline $\begin{array}{l}\text { Proceso de compra } \\
\text { no meditado }\end{array}$ & $\begin{array}{l}\text { Consumidor no analítico, } \\
\text { leal }\end{array}$ & $\begin{array}{l}\text { 1. Marca } \\
\text { 2. Costumbre } \\
\text { 3. Compras previas } \\
\text { 4. Referencias personales (boca a boca) }\end{array}$ \\
\hline $\begin{array}{l}\text { Proceso de compra } \\
\text { meditado y analítico }\end{array}$ & Consumidor analítico & $\begin{array}{l}\text { 1. Precio } \\
\text { 2. Calidad } \\
\text { 3. Sabor } \\
\text { 4. Calorías }\end{array}$ \\
\hline $\begin{array}{l}\text { Proceso de compra } \\
\text { crítico y saludable }\end{array}$ & Consumidor "consternado" & $\begin{array}{l}\text { 1. Calidad } \\
\text { 2. No utilización de químicos } \\
\text { 3. Frescura de productos } \\
\text { 4. Ingredientes } \\
\text { 5. Light u orgánicos }\end{array}$ \\
\hline
\end{tabular}

Fuente: Las entrevistas

En general, a mayor análisis en el proceso de compra, mayor es la creencia de que la medida puede ayudar a reducir el consumo de productos no saludables.

\section{Conocimiento acerca del sistema "semáforo" de alertas en el etiquetado}

En general los entrevistados muestran un alto nivel de conocimiento acerca del tema ya que en su mayoría habían leído en los diarios o escuchado en televisión o radio al respecto. Apenas un 10 por ciento manifestó no conocer absolutamente nada sobre el tema.

\section{Impacto en el consumo del sistema "semáforo" de alertas en el etiquetado}

Los participantes aceptaron que continuaban comprando los mismos productos y marcas pero reduciendo el consumo de aquellos productos con alto contenido de azúcar, sal y grasas. Al solicitar a los entrevistados que personifique a los productos alimenticios con alto nivel de grasa, azúcar o sal describiendo como sería físicamente y cuál sería su personalidad, las respuestas, ordenadas por frecuencia de mención, fueron las siguientes: 
- Gordo

- Enfermo

- Cerdo

- Con problemas cardiacos

Otras respuestas con menor frecuencia incluyeron frases o palabras tales como:

- Persona débil

- Persona con malos hábitos

- Falta de cuidado

- Adicto

- Descuidado/despreocupado

Se observa claramente una asociación de los productos con alto contenido en grasa azúcar y sal mayormente con características estética y medicamente indeseables como la gordura y el exceso de peso. Sin embargo, los entrevistados también asociaron a este tipo de productos con enfermedades sobre todo con problemas cardiacos, de circulación etc. Aunque algunas respuestas fueron variadas, todas coinciden en ser atributos y características negativas e indeseables en una persona.

Al ser consultados sobre si algún producto le había sorprendido por su etiqueta, los entrevistados manifestaron una serie de productos que se detallan en la siguiente tabla:

\section{Tabla 2}

\section{Productos cuyas etiquetas causaron sorpresa a los entrevistados}

\begin{tabular}{|l|l|}
\hline \multicolumn{1}{|c|}{ Producto } & \multicolumn{1}{c|}{ Observación } \\
\hline Cola light & $\begin{array}{l}\text { Los entrevistados manifestaron no estar de acuerdo con que la etiqueta no tenga } \\
\text { alerta roja en cuanto a azúcar. Consideraron que aún si no tuviera azúcar la etiqueta } \\
\text { debería tener alguna alerta debido a la cantidad de químicos que contiene. }\end{array}$ \\
\hline Snacks & $\begin{array}{l}\text { La alerta debería ser mayor por cuanto estos productos son muy dañinos para los } \\
\text { niños }\end{array}$ \\
\hline Cereal integral & $\begin{array}{l}\text { Llamó la atención ya que tiene alto nivel de azúcar y se promociona como un } \\
\text { producto saludable. }\end{array}$ \\
\hline Queso & $\begin{array}{l}\text { También llamó la atención por ser considerado un producto bueno para la salud y } \\
\text { sin embargo, la etiqueta tiene alerta roja en cuanto a sal y grasas. }\end{array}$ \\
\hline
\end{tabular}

Fuente: Las entrevistas 
Finalmente, se planteó al entrevistado que escoja a qué lado del supermercado se iría si los productos con baja concentración de azúcar, sal y grasas estuviesen en el lado izquierdo y los productos de alto contenido estuviesen en el lado derecho y los resultados fueron los siguientes:

- Solo uno afirmó que iría a la sección con productos saludables. Los demás indicaron que asistirían a ambas secciones ya que hay productos que no pueden dejar de comprar.

- Admitieron que aunque les encantaría dejar de consumir productos con etiqueta roja hay productos que no pueden dejar de consumir y que lastimosamente no se ofrecen en versiones "light" o con menor contenido de grasa, azúcar o sal.

- Aquellos con hijos más pequeños (entre los 5 y los 12 años) afirmaron que en los pocos casos en los cuales encuentran un producto sustituto más saludable, se ven obligados a seguir comprando el producto con etiqueta roja ya que el sabor no es tan agradable.

\section{Posición frente a la medida}

Los entrevistados manifestaron estar a favor de la medida e indicaron estar de acuerdo con que las autoridades implementen este tipo de acciones. Sin embargo, se mostraron muy escépticos en cuanto a que la medida logre un impacto considerable debido a:

Politización del tema: en vez de analizarse la medida de forma objetiva, se debate y polemiza en cuanto al impacto al sector empresarial y la libertad del consumidor a elegir o inclusive el partido político que propone la medida.

... creo que las personas nunca estamos de acuerdo y no nos acoplamos. Nos quejamos de todo y por quejarnos (Padre de familia).

Factores culturales: las personas continúan y continuarán comprando los productos así sean dañinos para la salud ya que no se le da importancia a la adecuada nutrición.

... Es lo mismo que el cigarrillo, la gente ve en la etiqueta fotos horribles, tiene que pagar impuesto por el cigarrillo pero igual fuman (Madre de familia). 
Falta de opciones saludables: Aun cuando los consumidores observan las alertas en las etiquetas de los productos, no encuentran una marca que ofrezca un producto similar pero más saludable. Los productos saludables suelen ser de un precio más elevado lo cual dificulta su compra.

El problema es que lo sano es más caro. Ejemplo: La cola cuesta un dólar y medio los tres litros cuando un jugo cuesta aproximadamente dos dólares el cartón de un solo litro (Padre de familia).

Falta de capacitación a la ciudadanía: Si bien los productos tienen las alertas, muchos desconocen lo que significan realmente o lo que estos productos están causando a su organismo por lo que es necesario una campaña de concientización

\section{Discusión}

El estudio muestra que si bien los entrevistados se muestran escépticos en cuanto a que la medida adoptada pueda cambiar considerablemente los malos hábitos alimenticios de la población ecuatoriana, la mayoría manifiesta haber reducido el consumo de productos con alto contenido de azúcar, sal y grasa disminuyendo las porciones y la frecuencia de consumo. Sin embargo, los padres de familia reconocen que no podrán eliminar por completo el consumo de estos productos por lo que seguirán adquiriéndolos. Las acciones gubernamentales por tanto, no solo deberían enfocarse en el consumidor, sino también en lograr que los productores mejoren la composición de sus productos instando a las empresas productoras a disminuir la cantidad de azúcar, sal y grasas que contienen a través de algún programa de incentivos que motive a las empresas a tener productos en niveles de alerta verde.

La investigación permitió determinar que el impacto de la medida también depende del proceso de compra que lleva a cabo el consumidor ecuatoriano, habiéndose identificado tres tipos diferentes de procesos de acuerdo al tiempo, el nivel de análisis y reflexión que lleva a cabo el comprador al momento de realizar la compra. El proceso de compra no meditado tiene como principales factores la marca y la experiencia previa que tiene el consumidor con esta mientras el proceso de compra meditado y analítico engloba distintos factores como precio, calidad, sabor y calorías que contiene el producto. En el proceso de compra "crítico y saludable" el comprador em- 
plea gran cantidad de tiempo y reflexión para adquirir los mejores productos en cuanto a sus beneficios para la salud, valorando la calidad, frescura y ausencia de químicos por sobre los precios. El sistema de semáforo tiende a tener un impacto mayor en los compradores que llevan a cabo procesos de compra en el que incurren en mayor análisis y tiempo de reflexión.

En su mayoría los compradores entrevistados son conscientes de la existencia del sistema gráfico en las etiquetas de los productos que consumen y conocen a manera general que esta medida es para mejorar la salud de la población. Comprenden que la alerta "roja" corresponde a productos dañinos pero en algunos casos desconocen que impacto tiene en su salud el hecho de que el producto posea alto contenido de azúcar, sal o grasa. Las entidades gubernamentales, asociaciones sin fines de lucro y asociaciones de derechos del consumidor podrían aportar por tanto realizando campañas de comunicación enfocadas a crear una cultura de análisis, comparación y reflexión al momento de adquirir productos alimenticios, transformando así al comprador ecuatoriano en un cliente más crítico y exigente. En general la efectividad de la medida se ve afectada por factores culturales, la falta de opciones más saludables y de capacitación a la ciudadanía.

\section{Bibliografía}

Ayala, C., Tong, X., Valderrama, A., Ivy, A. \& Keenan, N. (2010) Actions taken to reduce sodium intake among adults with self-reported hypertension: Health Styles survey, 2005 and 2008. J Clin Hypertens (Greenwich), 12(10), 793-9.

Feunekes, G. I., Gortemaker, I. A., Willems, A. A., Lion, R., \& Van Den Kommer, M. (2008). Front-of-pack nutrition labelling: testing effectiveness of different nutrition labelling formats front-of-pack in four European countries. Appetite, 50(1), 57-70.

Grimes, C.A., Riddell, L.J., \& Nowson, C.A. (2009). Consumer knowledge and attitudes to salt intake and labelled salt information. Appetite, 53(2),18994.

Heimbach, J.T. (1985). Sodium, hypertension and the American public: second tracking survey. Public Health Rep, 100(4), 371-2.

Kelly, B, Hughes, C., Chapman, K., Louie, J. CY., Dixon, H., Crawford, J., et al. (2009). Consumer testing of the acceptability and effectiveness of front- 
of-pack food labelling systems for the Australian grocery market. Health Promot Int., 24(2), 120-9.

Mhurchu, C., \& Gorton, D. (2007). Nutrition labels and claims in New Zeland and Australia: a review of use and understanding. Australian and New Zealand. Journal of Public Health, 31(2), 105-112.

Ministerio de Salud Pública (2013). Reglamento sanitario de etiquetado de alimentos procesados para el consumo humano. Acuerdo Ministerial \#4522.

(2014). Reglamento sanitario sustitutivo de etiquetado de alimentos procesados para el consumo humano. Registro Oficial, segundo suplemento.

Ortiz, M. (2014). ¡Ecuador aprueba el semáforo en el etiquetado de alimentos! Blog de Consumers International. Recuperado de: http://consumersinternational-es.blogspot.com/2014/06/ ecuador-aprueba-el-semaforo-en-el.html

Orozco, M. (2014). Cuatro cambios a la norma sobre etiquetado. Recuperado de http://www.elcomercio.com/actualidad/negocios/cuatro-cambios-a-norma-etiquetado.html.

Sanz-Valero, J., Sebastián-Ponce, M.I., \& Wanden-Berghe, C. (2012). Intervenciones para reducir el consumo de sal a través del etiquetado. Rev Panam Salud Pública, 31(4), 332-7. 